\title{
Correction to: The Effects of Gd-Free Impurity Phase on the Aging Behavior for the Microwave Surface Resistance of Ag-coated $\mathrm{GdBa}_{2} \mathrm{Cu}_{3} \mathrm{O}_{7-\delta}$ at Cryogenic Temperatures
}

\author{
Sungho Lee ${ }^{1} \cdot$ Woo II Yang ${ }^{1} \cdot$ Ho Sang Jung ${ }^{1,3} \cdot$ Won-Jae Oh ${ }^{2} \cdot$ Jiyeong Jang ${ }^{1} \cdot$ Jae-Hun Lee ${ }^{3} \cdot \mathrm{Kihyeok} \mathrm{Kang}^{3}$. \\ Seung-Hyun Moon ${ }^{3} \cdot$ Sang-Im Yoo ${ }^{2}$. Sang Young Lee ${ }^{1}$ (iD
}

Published online: 11 June 2018

@ The Korean Institute of Metals and Materials 2018

\section{Correction to: Electronic Materials Letters https://doi.org/10.1007/s13391-018-0068-4}

The original version of this article unfortunately contained a mistake. Figure 9 was incorrect. The corrected Fig. 9 is given below:
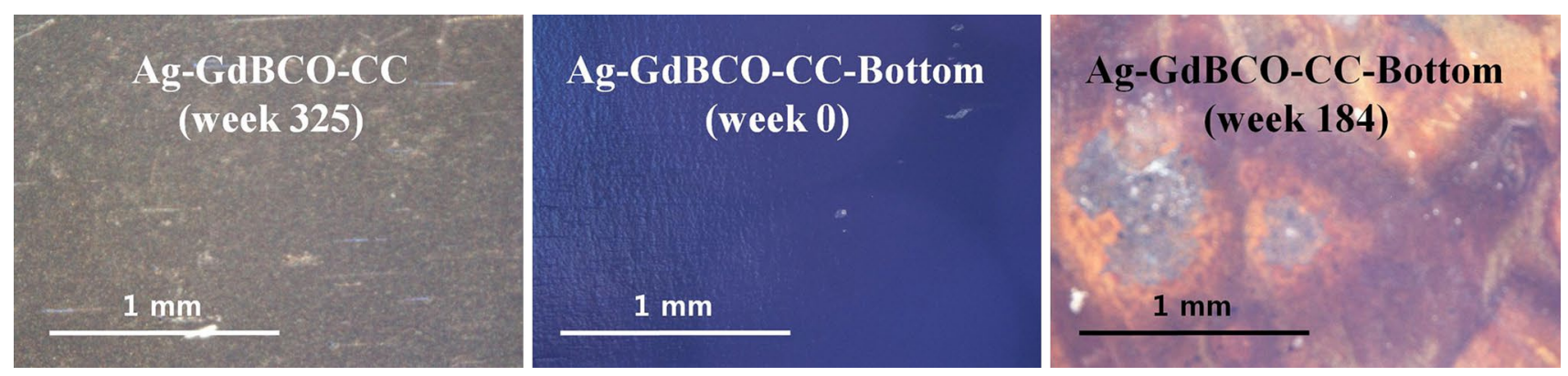

Fig. 9 Microscope pictures for (Left) a Ag-coated GdBCO CC aged 325 weeks, (Center) the bottom part of a pristine GdBCO layer, and (Right) the bottom part of a GdBCO layer aged 184 weeks after an
$600 \mathrm{~nm}$-thick Ag layer was deposited on top of the bottom part. All the pictures are magnified by 20 times

The original article can be found online at https://doi.org/10.1007/ s13391-018-0068-4.

Sang Young Lee

sylee@konkuk.ac.kr

1 Department of Physics, Konkuk University, Seoul 05029, Korea

2 Department of Materials Science and Engineering and Research Institute of Advanced Materials (RIAM), Seoul

National University, Seoul 08826, Korea

3 SuNAM Co., Ltd., Anseong, Gyunggi-do 17554, Korea 